\title{
Simultaneous enhancement of thermally activated delayed fluorescence and photoluminescence quantum yield via homoconjugation
}

\author{
Stephanie Montanaro ${ }^{+[a]}$ Piotr Pander, ${ }^{[b]}$ Mark R.J. Elsegood, ${ }^{[a]}$ Simon J. Teat, ${ }^{[c]}$ Andrew D. \\ Bond, ${ }^{[d]}$ lain A. Wright, ${ }^{*[a]}$ Daniel G. Congrave, ${ }^{*[d]}$ and Marc K. Etherington ${ }^{*[e]}$ \\ a. Department of Chemistry, Loughborough University, Epinal Way, LE11 3TU, United Kingdom, \\ E-mail: i.a.wright@lboro.ac.uk \\ b. Department of Physics, Durham University, South Road, Durham, DH1 3LE, United Kingdom \\ c. Advanced Light Source, Lawrence Berkeley National Laboratory, Cyclotron Road, Berkeley, CA, 94720, \\ USA \\ d. Yusuf Hamied Department of Chemistry, Cambridge, CB2 1EW, United Kingdom, E-mail: \\ dc704@cam.ac.uk \\ e. Department of Mathematics, Physics and Electrical Engineering, Northumbria University, Ellison Place, \\ Newcastle upon Tyne, NE1 8ST, United Kingdom, \\ E-mail: marc.k.etherington@northumbria.ac.uk
}

\begin{abstract}
A fundamental problem facing thermally activated delayed fluorescence (TADF) is to overcome the paradox of efficient electronic transitions and a narrow singlet-triplet energy gap $\left(\Delta E_{S T}\right)$ in a single luminophore. We present a quinoxaline-based TADF iptycene as the first clear example that homoconjugation can be harnessed as a viable design strategy toward this objective. Homoconjugation was introduced in an established TADF luminophore by trimerization through an iptycene core. This homoconjugation was confirmed by electrochemistry. As a direct consequence of homoconjugation we observed synergistic improvements to photoluminescence quantum yield $\left(\Phi_{\mathrm{PL}}\right)$, radiative rate of singlet decay $\left(\mathrm{k}_{r}^{\mathrm{S}}\right)$, delayed fluorescence lifetime (TTADF), and rate of reverse intersystem crossing $\left(\mathrm{k}_{\mathrm{rlSC}}\right)$, while narrowing the $\Delta E_{\mathrm{ST}}$. The cooperative enhancement is rationalised with TD-DFT calculations including spin-orbit coupling (SOC). A facile synthesis of this system, and the ubiquity of the pyrazine motif in state-of-the-art TADF materials across the electromagnetic spectrum, leads to a great potential for generality.
\end{abstract}

\section{Introduction}

Materials that exhibit thermally activated delayed fluorescence (TADF) are heralded as next generation emitters for organic light-emitting diodes (OLEDs). TADF materials require small singlet-triplet energy gaps $\left(\Delta E_{\mathrm{ST}}\right)$ to enable triplet harvesting, which is typically achieved through intramolecular charge transfer (ICT) states. ${ }^{1-3}$ Unfortunately, reducing the spatial overlap of the electron and hole wavefunctions causes a significant reduction in oscillator strength. Thus, a rapid reverse intersystem crossing (rISC) rate ( $\mathrm{k}_{\mathrm{rISC}}$ ) often comes at the expense of a high photoluminescence quantum yield (PLQY, $\Phi_{\mathrm{PL}}$ ) and a fast radiative rate $\left(k_{r}\right) .{ }^{4}$ For highly efficient and stable OLEDs the combination of high PLQY and large $\mathrm{k}_{\mathrm{r} I S c}$ is prized. ${ }^{5-9}$ Hence, new molecular design concepts to synergistically enhance PLQY and $k_{r}$ alongside $\mathrm{k}_{\mathrm{r} I \mathrm{sc}}$ are of vital importance. 
To obtain efficient rISC, a narrow $\Delta E_{\mathrm{ST}}$ is required. ${ }^{3} \mathrm{~A}$ large spin-orbit coupling is also essential, and is achieved through introducing a manifold of partially delocalised triplet states of both locally-excited (LE) and charge-transfer (CT) character. ${ }^{3,6,9,10}$ Structurally, this has translated to ICT molecules built up of numerous weakly coupled chromophores, conjugated through twisted bonds and/or cross conjugation. Prominent examples are 5Cz-TRZ and TAT$3^{3 \mathrm{DBTO}_{2}}$ reported by Cui, Chen, Adachi and Friend et al. ${ }^{6}$ and by Bryce, Monkman and coworkers, ${ }^{5}$ respectively (Figure $\left.1 \mathrm{a}\right)$.

Homoconjugation (through-space conjugation) is a weak form of electronic communication distinct from conventional through-bond conjugation. ${ }^{11-14}$ Homoconjugation has recently received significant attention, mediating communication between donor and acceptor moieties to introduce efficient through-space charge-transfer-TADF (TSCT-TADF), ${ }^{15-26}$ including triptycene-based structures. ${ }^{27-31}$ Triptycene is an archetypal homoconjugated hydrocarbon. ${ }^{32-37}$ The $\pi$-systems of the three "fins" are separated by the saturated $\mathrm{sp}^{3}$ bridgehead carbon and cannot communicate through-bond. However, the rigid conformation of triptycene facilitates trans-annular through-space overlap between their $\pi$-systems (Figure 1b).

A promising phenomenon attributed to homoconjugation in symmetrical iptycenes is an enhancement of $\pi-\pi^{*}$ oscillator strength due to cooperative through-space interactions between chromophores. Experimentally this is observed as a significantly greater than factor of three increase in extinction coefficient $(\varepsilon)$ compared with a single fin congener. ${ }^{38-40}$ Recently, we extended this phenomenon to ICT transitions. ${ }^{41}$ Upon trimerization of a fluorescent luminophore through a homoconjugated iptycene core, large enhancements in ICT parameters such as $\varepsilon, \mathrm{k}_{\mathrm{r}}$, and PLQY (i.e. radiative transitions) were observed.

In this work we will exploit two precedents: 1) the increase of TADF efficiency afforded by weakly coupling numerous chromophores, ${ }^{5,6}$ and 2 ) the enhancement of ICT radiative processes via homoconjugation. ${ }^{38-41}$ This allows the improvement of the radiative decay and $\mathrm{k}_{\mathrm{rISC}}$ of a TADF luminophore simultaneously.
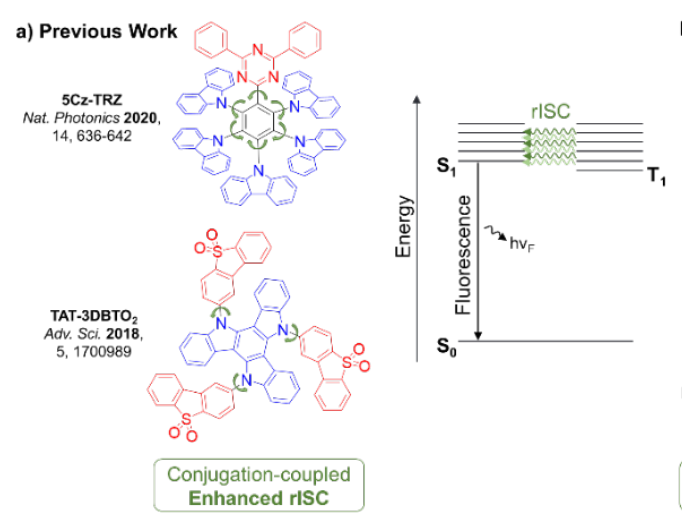

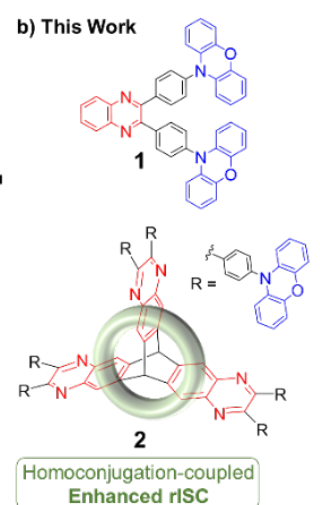

Enhanced rISC

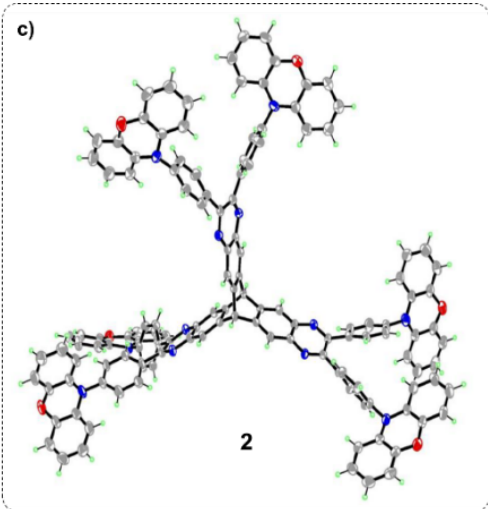


Figure 1. a) Previous and b) new designs for enhancing TADF through weakly coupled components; c) X-ray crystal structure of $2,7 \mathrm{CHCl}_{3}$ of crystallisation omitted for clarity.

\section{Results and discussion}

\section{Design and synthesis}

To test our hypothesis the iptycene 2 was synthesised. Three factors led to the selection of this system: 1) The opportunity for comparison with the homologous TADF fin $1,{ }^{42} 2$ ) the facile synthesis (Scheme S2.1) of quinoxaline-based iptycenes, and 3) the ubiquity of the pyrazine motif in TADF materials. ${ }^{43-49}$ The NMR characterisation of the compounds can be found in the supplementary information (Figures S3.1-S3.5). The structure of $2 \cdot 7 \mathrm{CHCl}_{3}$ was confirmed by single crystal X-ray diffraction (Figures 1c and S4.7 and Table S4.3). 2 crystallised as a $\mathrm{CHCl}_{3}$ heptasolvate, presumably due to the large internal free volume of iptycene structures. ${ }^{50}$ The structure of two, polymorphic, $\mathrm{CDCl}_{3}$ mono-solvates of 1 were also determined using synchrotron radiation (Tables S4.1-S4.2 and Figures S4.1-S4.6).t

\section{Computational chemistry}

1 and 2 were investigated using TD-PBE0/def2svp (Figure 2, Figures S6.1-S6.34). ${ }^{51}$ The lowest energy singlet and triplet states for $\mathbf{1}$ and $\mathbf{2}$ are predominantly represented by ICT transitions between the phenoxazine donor and quinoxaline acceptor moieties involving the HOMO and LUMO as expected (Figures S6.1, S6.3, S6.6, S6.9, S6.33 and S6.34). ${ }^{42}$ For 2 the $S_{1}$ and $S_{2}$ states are near-degenerate (Figure 2a) - a consequence of the localisation of the LUMO/ LUMO+1 orbitals of the iptycene core of 2 due to through-space $\pi$-system overlap between fins. Such a MO structure is in agreement with established experimental data for triptycene, ${ }^{48,52,53}$ and indicates that homoconjugation should influence the photophysical properties of 2. Narrow $\Delta E_{S T}$ values are predicted for 1 and 2 (0.01 and $0.03 \mathrm{eV}$, respectively). Calculations predict a notably larger oscillator strength $(f)$ for the $S_{0} \rightarrow S_{1}$ transition of 2 than for 1 ( $f=0.051$ vs. 0.003). $f$ is similarly large for the near-degenerate $S_{0} \rightarrow S_{2}$ transition of 2 $(f=0.051)$. Larger $f$ for the lowest lying singlet states of 2 compared with 1 are promising for enhancements in $\varepsilon$ and the radiative rate of singlet decay $\left(k_{r}{ }^{S}\right)$ (confirmed experimentally below). Furthermore, within $<0.4 \mathrm{eV}$ of $\mathrm{T}_{1}$ a manifold of 26 higher energy singlet and triplet states are predicted for the iptycene 2 (Figures S6.7-S6.32), in contrast to only 4 for 1 (Figures S6.2-S6.5). This collection of energetically close excited states (Figure 2b), comprising numerous CT states spread across the different fins of $\mathbf{2}$ and a larger number of ${ }^{3}$ LE states than for $\mathbf{1}$, is highly encouraging for accelerated delayed fluorescence in $\mathbf{2}$. ${ }^{5,6,9}$

Spin-orbit coupling (SOC) is essential to permit the formally forbidden spin-flip associated with rISC. Hence, the merit of the manifold of triplet states predicted for $\mathbf{2}$ was investigated 
by calculating the spin-orbit coupling matrix elements (SOCMEs) for the ground state optimized structure (TD-PBE0/def2svp and PySOC script ${ }^{54}$ ). The relevant states were probed via electron-hole analysis with the Multiwfn program to determine their CT or LE character. ${ }^{55-}$ ${ }^{57}$ Detailed electron-hole analysis and assignment of excited state character are presented in the ESI (section 6, Table S6.1). Some relevant natural transition orbitals (NTOs) for 2 are shown in Figure 2c. The fins of 2 in Figure $2 c$ are denoted $A, B$ and $C$ to aid discussion of the spatial distribution of states. Data for $S_{1}, T_{1}$, and triplet states with SOCME $>0.1 \mathrm{~cm}^{-1}$ to $S_{1}$ within $<0.4 \mathrm{eV}$ of $\mathrm{T}_{1}$ are summarized in Table 1 for 1 and 2.

In agreement with what is intuitive upon visual inspection of the NTOs (Figure 2a and c), the $S_{1}$ state of 2 is assigned as CT in nature. The electron-hole analysis for $T_{1}$ is near-identical to that of $S_{1}$, meaning both states are the same in configuration (CT) with the same spatial distribution. Hence, as expected from El-Sayed's rule ${ }^{58}$ the calculated $S_{1}-T_{1}$ SOCME is very small $\left(0.05 \mathrm{~cm}^{-1}\right)$, and rISC should be very inefficient between these states. A similar case is observed for the $S_{1}$ and $T_{1}$ states of 1 (Table 1, Figures S6.1 and S6.3). 

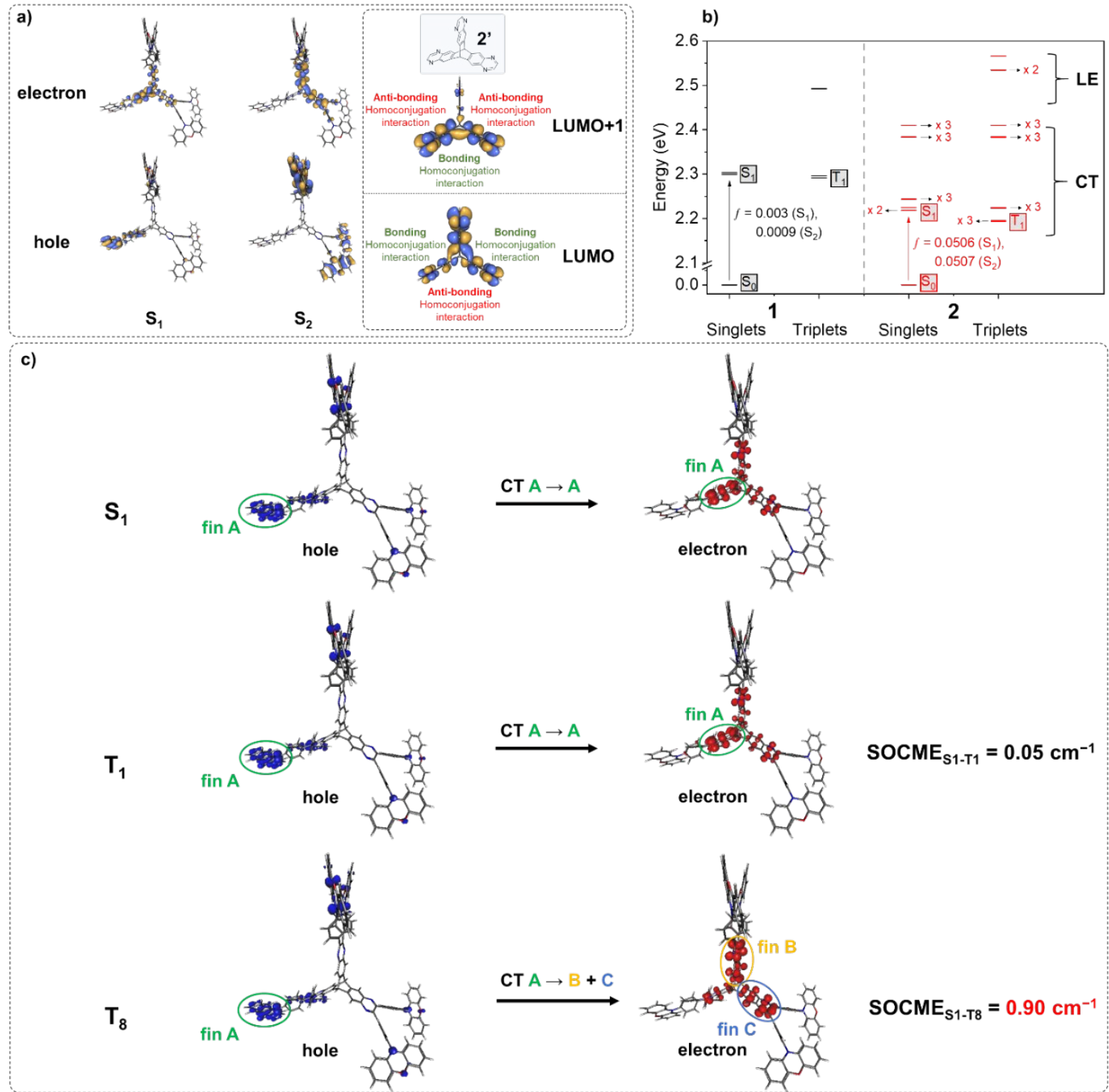

Figure 2. a) Natural transition orbitals for $S_{1}$ and $S_{2}$ states of 2. The LUMO and LUMO+1 orbitals of the core 2' are included to highlight the effect of homoconjugation within the LUMO manifold; b) Jablonski diagram for 1 and 2 ; c) Natural transition orbitals for the $S_{1}$ and appropriate $T$ states of 2 to highlight spin-orbit coupling.

Table 1. Summary of computational results. 


\begin{tabular}{|c|c|c|c|c|}
\hline Compound & State & $\begin{array}{c}\Delta \mathrm{E}_{\mathrm{s} 1-\mathrm{Tn}} / \\
\mathrm{eV}^{\mathrm{a}}\end{array}$ & $\begin{array}{c}\text { SOCMES1-Tnl } \\
\mathrm{cm}^{-1 \mathrm{~b}}\end{array}$ & Assignment $^{c}$ \\
\hline & $\mathrm{S}_{1}$ & - & - & CT \\
\hline \multirow[t]{5}{*}{1} & $\mathrm{~T}_{1}$ & 0.01 & 0.05 & CT \\
\hline & $\mathrm{T}_{3}$ & -0.19 & 0.23 & LE \\
\hline & $\mathrm{S}_{1}$ & - & - & $\mathrm{CT} A \rightarrow \mathrm{A}$ \\
\hline & $\mathrm{T}_{1}$ & 0.03 & 0.05 & $\mathrm{CT} A \rightarrow \mathrm{A}$ \\
\hline & $\mathrm{T}_{7}$ & -0.17 & 0.29 & $\mathrm{CT} \mathrm{B}+\mathrm{C} \rightarrow \mathrm{A}$ \\
\hline \multirow[t]{4}{*}{2} & $\mathrm{~T}_{8}$ & -0.17 & 0.90 & CT $\mathrm{A} \rightarrow \mathrm{B}+\mathrm{C}$ \\
\hline & $\mathrm{T}_{13}$ & -0.32 & 0.11 & LE \\
\hline & $\mathrm{T}_{14}$ & -0.32 & 0.08 & LE \\
\hline & $\mathrm{T}_{15}$ & -0.35 & 0.12 & LE \\
\hline
\end{tabular}

${ }^{a}$ Calculated energy gap between $S_{1}$ and $T_{n}$; ${ }^{b}$ Calculated spin-orbit coupling matrix element between $S_{1}$ and $T_{n}$; ${ }^{\mathrm{C}} \mathrm{A}, \mathrm{B}$ and $\mathrm{C}$ fin nomenclature is consisted with Figure $2 \mathrm{c} ; \mathrm{CT}=$ charge transfer, $\mathrm{LE}=$ locally excited.

It has been accepted that in TADF materials vibronic coupling can facilitate rISC between a CT singlet such as $S_{1}$ and upper LE triplet states, for which there should be greater SOC due to a change in excited state configuration. ${ }^{3,6,8-10}$ Indeed, the SOCME values between $\mathrm{S}_{1}$ and upper LE triplet states ( $T_{3}$ for $1 ; T_{13}, T_{14}$ and $T_{15}$ for 2 ) are on the order of $0.1-0.25 \mathrm{~cm}^{-1}$.

We note for 1 that there is only one LE triplet state $\left(T_{3}\right)$ within $0.4 \mathrm{eV}$ of $T_{1}$ with a greater SOCME to $S_{1}$ than $T_{1}$. This is in stark contrast to what is observed for 2 .

We can explain the more efficient rISC observed experimentally for 2 through the larger presence of states with high SOC. Firstly, 2 has three LE triplets ( $T_{13}, T_{14}$ and $\left.T_{15}\right)$ that exhibit appreciable SOCMEs with $S_{1}$. Secondly, due to electronic coupling between the fins of 2 , it is possible to localise CT states across neighbouring fins (Figure $2 \mathrm{c}$ ). This is apparent for the $T_{8}$ state which is localised differently to $S_{1} . T_{8}$ is a CT state with the hole predominantly localised on the phenoxazine moieties of fin $A$, and the electron mainly on the quinoxaline heterocycles of fins $B$ and $C$ with some bridging $A$ contribution. Conversely, for $S_{1}$ both the electron and hole are predominantly localised over fin A with some electron density extending onto the quinoxalines of $B$ and $C$. These distinct differences in the localisation of $S_{1}$ and $T_{8}$, can translate into a change in orbital angular momentum between the two states, but they crucially retain some spatial overlap over the homoconjugated core of $\mathbf{2}$. This enables a large SOCME value of $0.9 \mathrm{~cm}^{-1}$ between the CT states $S_{1}$ and $T_{8}$, which are predicted to be only $0.17 \mathrm{eV}$ apart in energy (Table 1). A significant SOCME is also predicted between $\mathrm{S}_{1}$ and $\mathrm{T}_{7}$ $\left(0.29 \mathrm{~cm}^{-1}\right)$, which is a CT state degenerate with $\mathrm{T}_{8}$ also spread across multiple fins (Table 1, Figure S6.18).

While the exact rISC mechanism in $\mathbf{2}$ may be complex, from simply considering the SOCMEs calculations make it abundantly clear that homoconjugation in $\mathbf{2}$ can facilitate strong SOC 
interactions between TADF-relevant states, which is not predicted for the non-homoconjugated single chromophore 1.

\section{Electrochemistry}

1 and 2 display identical first oxidation potentials ( $\left.E^{o x}\right)$ (Figure 3, Table S5.1), consistent with localisation of the HOMO of $\mathbf{2}$ on the peripheral phenoxazine donors. Conversely, $\mathbf{2}$ displays a less negative first reduction potential $\left(E^{\text {red }}\right)$ than $\mathbf{1}$, and hence a more accessible LUMO, ascribed to electronic communication across the iptycene core of 2 , which increases the acceptor strength. Additionally, while the first reduction wave for $\mathbf{1}$ corresponds to what is typically expected for a single electrochemically reversible process, the wave for $\mathbf{2}$ is clearly a superposition of three electrochemical reductions. This electrochemical behavior supports weak electronic coupling between the multiple fins of 2 through the lower lying unoccupied molecular orbitals, consistent with homoconjugation and the DFT predictions above.
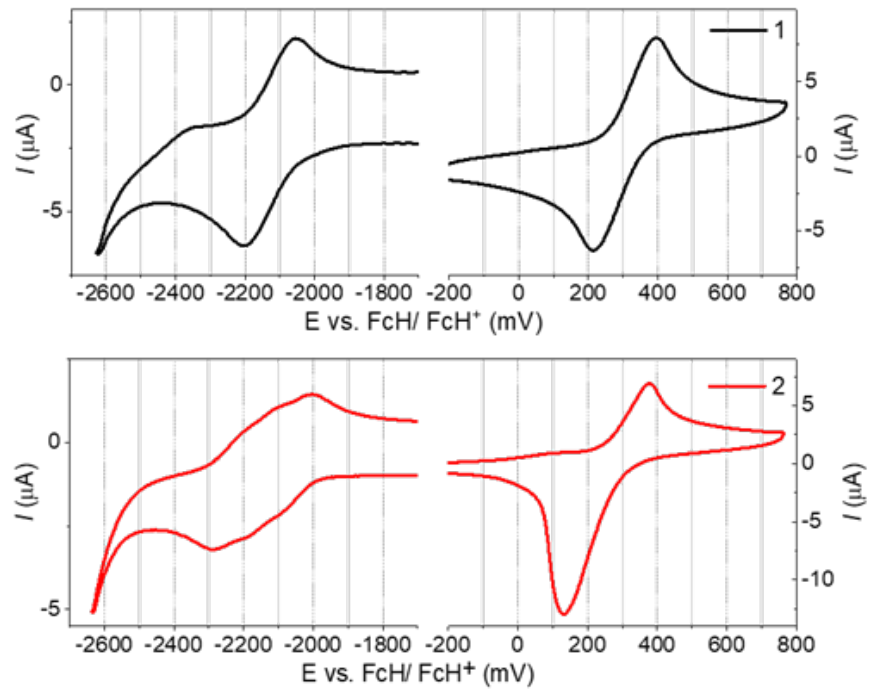

Figure 3. Cyclic voltammograms for 1 and 2 measured in $0.1 \mathrm{M} \mathrm{n}$-Bu4 $\mathrm{PF}_{6}$ in 1,2-dichlorobenzene as the supporting electrolyte. Scan rate $=100 \mathrm{mV} \mathrm{s}^{-1}$.

\section{Photophysics}

Photophysical measurements (Table 2) in toluene solution (Figure 4) provide a platform to accurately compare pertinent rates, as the data can be considered intrinsic, with strictly monoexponential decay for both prompt and delayed fluorescence in the absence of any solid-state phenomena. The absorption (and PL) spectrum of $\mathbf{2}$ is bathochromically shifted compared with that of 1 due to trans-annular communication, consistent with electrochemical and TD-DFT results. The absorption spectra for 1 and 2 consist of intense transitions below ca. $350 \mathrm{~nm}$, assigned to $\pi-\Pi^{*}$ excitations, and lower energy broad ICT transitions $\geq 400 \mathrm{~nm}{ }^{59}$ Similarly, the PL spectra of 1 and 2 are also ascribed to ICT, being broad and featureless and exhibiting positive solvatochromism (Figure S8.1). 
As 2 can be considered a trimer of the monomer unit 1, the maximum ICT absorption extinction coefficient for $\mathbf{2}$ is expected to be three times that of $\mathbf{1}$ in the absence of any electronic communication between fins. However, our experimental values, obtained from triplicate measurements on rigorously dried samples, yield a ratio of 3.4 (Table 2) (for $1 \varepsilon=3.5 \times 10^{3} \mathrm{M}^{-1} \mathrm{~cm}^{-1}$ at $398 \mathrm{~nm}$; for $2 \varepsilon=12 \times 10^{3} \mathrm{M}^{-1} \mathrm{~cm}^{-1}$ at $420 \mathrm{~nm}$ ). An expansion of the ICT region of the UV-Vis spectra for 1 and $\mathbf{2}$ is provided in Figure S8.2. TD-DFT results indicate that the singlet ICT states of 2 have significant localisation over the homoconjugated iptycene core, while electrochemical measurements also indicate electronic communication between fins. Therefore, the larger than factor of three increase in ICT extinction coefficient for $\mathbf{2}$ compared to 1 is ascribed to a cooperative effect between the fins of 2 through homoconjugation. Concomitant with this increase, the solution values for $\Phi_{\mathrm{PL}}$ and $\mathrm{k}_{\mathrm{r}}{ }^{\mathrm{S}}$, are considerably enhanced for 2 compared with $1\left(1-\Phi_{\mathrm{PL}}=50 \%, \mathrm{k}_{\mathrm{r}}{ }^{\mathrm{S}}=1.8 \times\right.$ $\left.10^{7} \mathrm{~s}^{-1} ; 2-\Phi_{\mathrm{PL}}=63 \%, \mathrm{k}_{\mathrm{r}}^{\mathrm{S}}=2.4 \times 10^{7} \mathrm{~s}^{-1}\right)$. Notably, for 2 the value of $\mathrm{k}_{\mathrm{r}}^{\mathrm{s}}$ is increased by a third compared with 1 . Therefore, there is a clear enhancement in the probability of a radiative transition both to and from the ICT state in 2, crucially observed despite the narrower $\Delta E_{S T}$ of 2 compared with $1\left(1-\Delta E_{S T}=0.26 \mathrm{eV} ; 2-\Delta E_{\mathrm{ST}}=0.21 \mathrm{eV}\right.$ ), as measured in a zeonex host (Table 2, Figures S9.1S9.9 and S10.1). The acceleration of radiative transitions in $\mathbf{2}$ is very likely linked to homoconjugation, in-line with the computational predictions and electrochemical experiments above. Such an enhancement of ICT through homoconjugation has only been noted once previously, in a structure that does not display TADF. ${ }^{41}$ Notably, $\mathrm{S}_{1}$ is also significantly more stabilised than $\mathrm{T}_{1}$ upon trimerization of compound 1 to 2 , suggesting the iptycene core can be employed to fine-tune the $\Delta E_{\mathrm{ST}}$ of TADF compounds.

a)

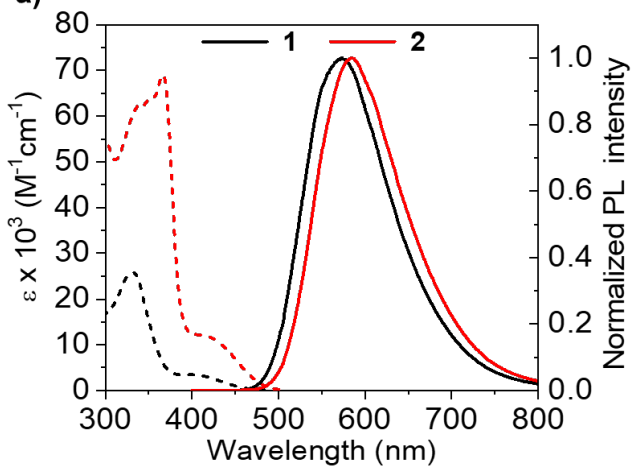

b)

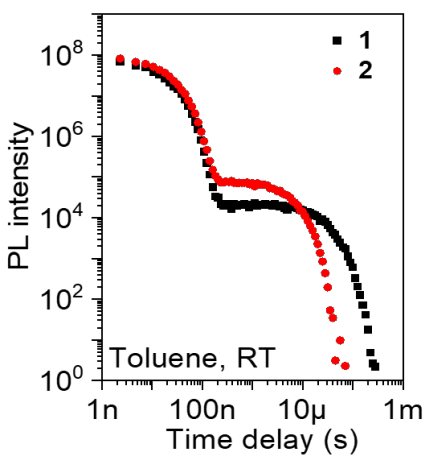

c)

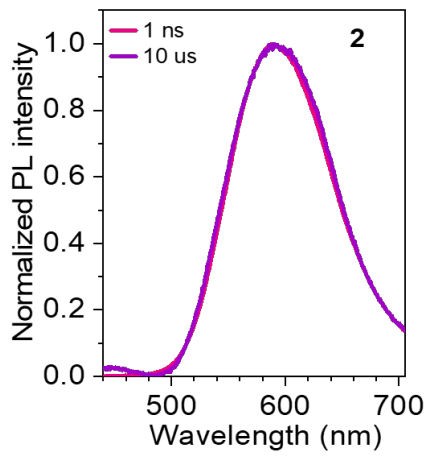

Figure 4. a) Extinction coefficient and steady state PL spectra $\left(1 \times 10^{-5} \mathrm{M}\right)$ of $\mathbf{1}$ and $\mathbf{2}$ in toluene; b) PL decays of $\mathbf{1}$ and $\mathbf{2}$ in degassed toluene; c) Prompt and delayed time-resolved spectra for $\mathbf{2}$ in degassed toluene.

Table 2. The key parameters obtained from the photophysical study of compounds 1 and 2. 


\begin{tabular}{|c|c|c|c|c|c|c|c|c|c|c|c|}
\hline & $\begin{array}{l}\text { Solvent I } \\
\text { matrix }\end{array}$ & $\begin{array}{c}\lambda_{\text {abs }} / \mathrm{nm} \\
{\left[\varepsilon \times 10^{3} \mathrm{M}^{-1} \mathrm{~cm}^{-1}\right]^{\mathrm{a}}}\end{array}$ & $\begin{array}{l}\lambda_{\mathrm{em}} / \\
\mathrm{nm}^{\mathrm{b}}\end{array}$ & $\begin{array}{c}\Delta E_{\mathrm{ST}} / \mathrm{eV} \\
{\left[\mathrm{S}_{1} / \mathrm{T}_{1}\right]^{\mathrm{c}}}\end{array}$ & $\Phi_{\mathrm{PL}}{ }^{\mathrm{d}}$ & $\Phi_{\mathrm{PF}}$ & $\Phi_{\mathrm{DF}}$ & $\begin{array}{c}\text { TPF } l \\
\text { ns }\end{array}$ & $\begin{array}{c}\text { TTADF } / \\
\mu \mathrm{S}\end{array}$ & $\begin{array}{c}\mathbf{k}_{\mathrm{r}}^{\mathbf{S}} / \\
10^{7} \mathrm{~s}^{-1} \\
\mathrm{e}\end{array}$ & $\begin{array}{l}k_{\text {RISc }} / \\
10^{4} \mathrm{~s}^{-1 \mathrm{f}}\end{array}$ \\
\hline \multirow{3}{*}{1} & Toluene & $\begin{array}{c}332[26], 398 \mathrm{sh} \\
{[3.5]}\end{array}$ & 574 & - & 0.50 & 0.35 & 0.15 & 19.6 & 27.3 & 1.8 & 2.5 \\
\hline & Zeonex & - & 504 & $\begin{array}{c}0.26 \\
{[2.74 / 2.48]}\end{array}$ & - & - & - & 8.9 & 656 & - & - \\
\hline & PVK:PBD & - & 548 & $\begin{array}{c}0.13 \\
{[2.54 / 2.41]}\end{array}$ & 0.63 & 0.41 & 0.22 & 16.4 & 26.2 & 2.5 & 3.4 \\
\hline \multirow{3}{*}{2} & Toluene & $\begin{array}{c}338 \text { [62], } 366 \text { [69], } \\
420 \mathrm{sh}[12]\end{array}$ & 583 & - & 0.61 & 0.48 & 0.13 & 20.5 & 5.8 & 2.4 & 8.7 \\
\hline & Zeonex & - & 517 & $\begin{array}{c}0.21 \\
{[2.65 / 2.44]}\end{array}$ & - & - & - & 12.4 & 90.1 & - & - \\
\hline & PVK:PBD & - & 556 & $\begin{array}{c}0.06 \\
{[2.47 / 2.41]}\end{array}$ & 0.75 & 0.49 & 0.26 & 24.0 & 16.7 & 2.1 & 6.5 \\
\hline
\end{tabular}

${ }^{a}$ sh = shoulder; ${ }^{b}$ steady state $\mathrm{PL}$ maxima; ${ }^{c}$ The $S_{1}$ energy is taken from delayed fluorescence (and not prompt fluorescence) spectra due to its relevance for the TADF mechanism; ${ }^{d}$ Total PLQY in degassed solution or

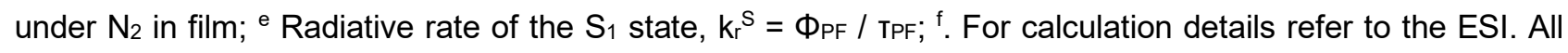
values determined at room temperature except for $T_{1}$ energy which is taken from phosphorescence spectra at $80 \mathrm{~K}$.

The TADF parameters of 1 and 2 were evaluated in toluene solution (Figure 4). Both luminophores exhibit a delayed fluorescence (DF) component that spectrally matches the prompt fluorescence (PF) (Figures 4c and S10.1) and contributes similarly to the total $\Phi_{\mathrm{PL}}(1$ - $\Phi_{\mathrm{DF}}=15 \% ; 2$ - $\Phi_{\mathrm{DF}}=13 \%$ ). However, the lifetime of the delayed fluorescence (TTADF) for the iptycene 2, is nearly five times shorter than that observed for 1 (Figure 4 b), in line with a greater than threefold increase in $\mathrm{k}_{\mathrm{rISC}}\left(1-\mathrm{TTADF}_{\mathrm{TA}}=27.3 \mu \mathrm{s}, \mathrm{k}_{\mathrm{rISC}}=2.5 \times 10^{4} \mathrm{~s}^{-1} ; 2\right.$ - TTADF $=5.8$ $\mu \mathrm{s}, \mathrm{k}_{\mathrm{rlsc}}=8.7 \times 10^{4} \mathrm{~s}^{-1}$ ). This is consistent with the dense manifold of energetically close excited states and enhanced SOC in 2 predicted by TD-DFT (Figure 2), a consequence of intrafin coupling via homoconjugation.

To summarise, considered alongside electrochemical and computational studies, the photophysical data obtained for $\mathbf{2}$ in solution indicate that it is possible to simultaneously amplify the radiative transition probability and $\mathrm{k}_{\mathrm{r} I S c}$ of a TADF luminophore by exploiting homoconjugation. Similar results are obtained in the PVK:PBD blend used for OLED fabrication below i.e. a larger PLQY, shorter $\mathrm{TTADF}_{\mathrm{TA}}$, and faster $\mathrm{k}_{\mathrm{rISC}}$ for 2 compared with 1 (Figures S11.1-S11.6)

\section{Electroluminescence}


Solution-processed OLED devices were fabricated using 1 and 2 as emitters, denoted Dev 1 and Dev 2, respectively. Pertinent data and the device structure are shown in Figure 5. Additional data are presented in the supporting information (Figure S12.1, Table S12.1). The device structure offers low $\mathrm{V}_{\mathrm{ON}}$ and large current densities, desirable for high device luminance, but also high external quantum efficiency (EQE) due to the presence of an additional electron blocking PVKH layer. ${ }^{60}$ Dev 1 and Dev 2 display EQEs reaching maximum values of $9.7 \%$ and $11.9 \%$, respectively, in agreement with $\Phi_{\mathrm{PL}}$ in film, demonstrating a $\sim 20 \%$ increase in efficiency due to homoconjugation. Most crucially, Dev 2 displays smaller efficiency roll-off than Dev 1, thanks to the shorter TTADF and larger k $\mathrm{rISC}_{\text {of }} 2$ in OLED host (Table 2). The difference in roll-off between Dev 1 and Dev 2 is manifested by their diverging characteristics of EQE vs. current density in Figure $5 \mathrm{c}$. The electroluminescence (EL) spectra match the PL in the same host (Table 2). The 11.9\% figure is larger than that reported earlier for a similar device structure. ${ }^{60}$. While the larger efficiency of Dev 2 contributes to its greater maximum luminance (Dev 1 - $8900 \mathrm{~cd} \mathrm{~m}^{-2}$; Dev 2 - $14800 \mathrm{~cd} \mathrm{~m}^{-2}$ ), the other significant factor is the higher current density (Dev $1-120$ mA cm${ }^{-2}$; Dev 2 - $220 \mathrm{~mA} \mathrm{~cm}^{-2}$ ). Both OLEDs show a similar $\mathrm{V}_{\mathrm{ON}}=5.5-6 \mathrm{~V}$ as 1 and 2 have nearly identical HOMO and LUMO energies.
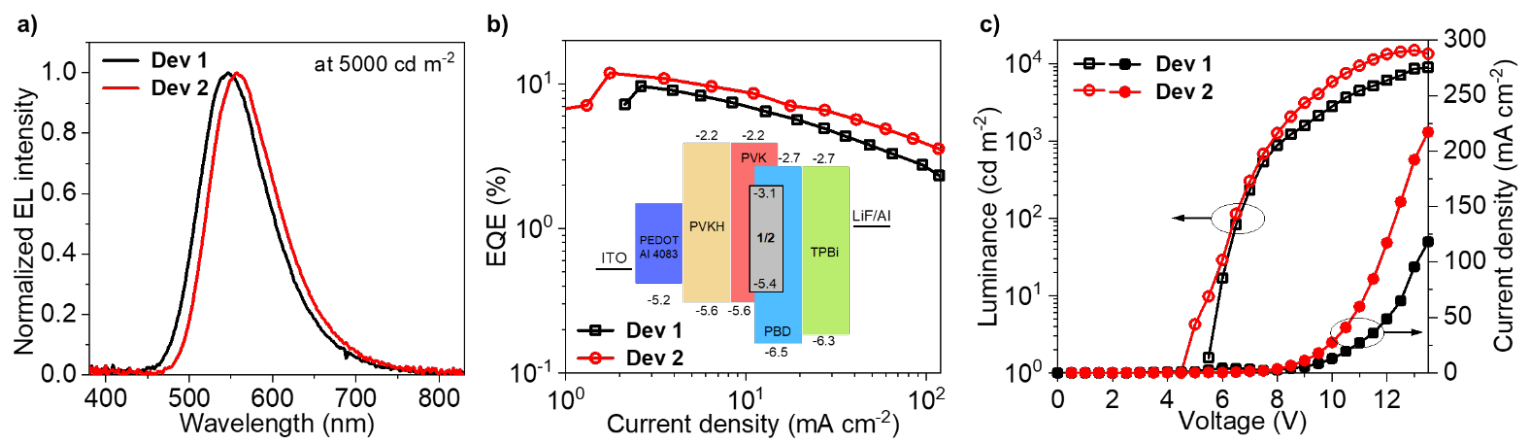

Figure 5. Electroluminescence characteristics for Dev 1 and Dev 2.

\section{Conclusions}

The simultaneous realization of high oscillator strength alongside efficient rISC is essential in the design of next generation TADF materials. For the first time we have proven that it is possible to harness homoconjugation as a viable strategy toward this objective. By trimerizing the known TADF luminophore 1 through an iptycene core, homoconjugation was induced in 2, which was observed electrochemically. As a direct consequence of the homoconjugation in $\mathbf{2}$, simultaneous improvements to PLQY, $\mathrm{k}_{r}{ }^{\mathrm{S}}, \mathrm{T}_{\mathrm{TADF}}, \mathrm{k}_{\mathrm{rISC}}$, and $\Delta E_{S T}$ were achieved, in-line with TD-DFT calculations which predicted a synergistic enhancement of oscillator strength $(f)$ and spin-orbit coupling (SOC). Owing to the facile synthesis of this new system, and the ubiquity of the pyrazine moiety in state-ofthe-art TADF materials across the electromagnetic spectrum from the deep blue to the near-infrared (NIR), ${ }^{43-48}$ this concept has great promise for generality. NIR TADF emitters in particular are poised to benefit from the enhancement in radiative transitions offered by homoconjugation and will be explored in future work. 


\section{Author Contributions}

S.M. Conceptualization, Formal analysis, Investigation, Writing - original draft, Writing - review \& editing, Visualization

P.P. Conceptualization, Formal analysis, Investigation, Writing - original draft, Writing - review \& editing, Visualization

M.R.J.E. Formal analysis (X-ray data sets), Investigation (X-ray Crystallography), Funding acquisition (ALS beam time), Validation (of the structures), Visualisation (X-ray structure figures), Writing - review \& editing S.J.T. Formal Analysis (X-ray data sets at the ALS)

A.D.B. Formal Analysis (X-ray data sets), Investigation (X-ray Crystallography), Validation (of the structures), Visualisation (X-ray structure figures).

I.A.W. Conceptualization, Funding acquisition, Project Administration, Supervision, Writing - review \& editing

D.G.C. Conceptualization, Project administration, Software, Visualization, Writing - original draft, Writing review \& editing;

M.K.E. Project Administration, Supervision, Writing - review \& editing

\section{Conflicts of interest}

There are no conflicts to declare.

\section{Acknowledgements}

S.M. thanks Loughborough University for a PhD Studentship. P.P. acknowledges the EPSRC (EP/S012788/1) for support. I.A.W. thanks the EPSRC (EP/T028688/1) and RSC (RF19-2751) for support. D.G.C. acknowledges the Herchel Smith fund for an early career fellowship, and $\mathrm{H}$. Bronstein (University of Cambridge) in a mentoring capacity and for providing laboratory space. This research used resources of the Advanced Light Source, which is a DOE Office of Science User Facility under contract no. DE-AC02-05CH11231. We would like to thank A. P. Monkman and F. B. Dias for access to their experimental setups. We thank Sobereva (USTB Beijing) for providing the Multiwfn program and providing instruction on how to run the software. We thank C. Zhong (Wuhan University) and W. Zheng (University of Cambridge) for help and advice with the PySOC script and running SOCME calculations.

\section{References}

f CCDC 2092859-61 contain the supplementary crystallographic data for this paper. These data can be obtained free of charge from The Cambridge Crystallographic Data Centre via www.ccdc.cam.ac.uk/structures.

1 H. Uoyama, K. Goushi, K. Shizu, H. Nomura and C. Adachi, Nature, 2012, 492, 234-238.

2 F. B. Dias, K. N. Bourdakos, V. Jankus, K. C. Moss, K. T. Kamtekar, V. Bhalla, J. Santos, M. R. Bryce and A. P. Monkman, Adv. Mater., 2013, 25, 3707-3714.

3 M. K. Etherington, J. Gibson, H. F. Higginbotham, T. J. Penfold and A. P. Monkman, Nat. Commun., 2016, 7, 13680.

4 B. Wex and B. R. Kaafarani, J. Mater. Chem. C, 2017, 5, 8622-8653. 
P. L. dos Santos, J. S. Ward, D. G. Congrave, A. S. Batsanov, J. Eng, J. E. Stacey, T. J. Penfold, A. P. Monkman and M. R. Bryce, Adv. Sci., 2018, 1700989.

L. S. Cui, A. J. Gillett, S. F. Zhang, H. Ye, Y. Liu, X. K. Chen, Z. Sen Lin, E. W. Evans, W. K. Myers, T. K. Ronson, H. Nakanotani, S. Reineke, J. L. Bredas, C. Adachi and R. H. Friend, Nat. Photonics, 2020, 14, 636-642.

X. K. Chen, Y. Tsuchiya, Y. Ishikawa, C. Zhong, C. Adachi and J. L. Brédas, Adv. Mater., 2017, 29, $1-8$.

X. K. Chen, S. F. Zhang, J. X. Fan and A. M. Ren, J. Phys. Chem. C, 2015, 119, 9728-9733.

H. Noda, X. K. Chen, H. Nakanotani, T. Hosokai, M. Miyajima, N. Notsuka, Y. Kashima, J. L. Brédas and C. Adachi, Nat. Mater., 2019, 18, 1084-1090.

J. Gibson, A. P. Monkman and T. J. Penfold, ChemPhysChem, 2016, 17, 2956-2961.

J. Li, P. Shen, Z. Zhao and B. Z. Tang, CCS Chem., 2019, 1, 181-196.

L. T. Scott, Pure Appl. Chem., 1986, 58, 105-110.

A. Braendle, A. Perevedentsev, N. J. Cheetham, P. N. Stavrinou, J. A. Schachner, N. C. MöschZanetti, M. Niederberger and W. R. Caseri, J. Polym. Sci. Part B Polym. Phys., 2017, 55, 707-720.

L. T. Scott and M. M. Hashemi, Tetrahedron, 1986, 42, 1823-1830. Angew. Chemie Int. Ed., 2021, anie.202109041.

K.-L. L. Woon, C.-L. L. Yi, K.-C. C. Pan, M. K. Etherington, C.-C. C. Wu, K.-T. T. Wong and A. P. Monkman, J. Phys. Chem. C, 2019, 123, 12400-12410.

Y. Wang, C. Huang, H. Ye, C. Zhong, A. Khan, S. Yang, M. Fung, Z. Jiang, C. Adachi and L. Liao, Adv. Opt. Mater., 2020, 8, 1901150.

X. Tang, L.-S. Cui, H.-C. Li, A. J. Gillett, F. Auras, Y.-K. Qu, C. Zhong, S. T. E. Jones, Z.-Q. Jiang, R.

H. Friend and L.-S. Liao, Nat. Mater., 2020, 19, 1332-1338.

H. Tsujimoto, D.-G. Ha, G. Markopoulos, H. S. Chae, M. A. Baldo and T. M. Swager, J. Am. Chem. Soc., 2017, 139, 4894-4900.

S.-Y. Yang, Y.-K. Wang, C.-C. Peng, Z.-G. Wu, S. Yuan, Y.-J. Yu, H. Li, T.-T. Wang, H.-C. Li, Y.-X. Zheng, Z.-Q. Jiang and L.-S. Liao, J. Am. Chem. Soc., 2020, 142, 17756-17765.

X. Wang, S. Wang, J. Lv, S. Shao, L. Wang, X. Jing and F. Wang, Chem. Sci., 2019, 10, 2915-2923.

Q. Li, J. Hu, J. Lv, X. Wang, S. Shao, L. Wang, X. Jing and F. Wang, Angew. Chemie Int. Ed., 2020, 59, 20174-20182.

X.-L. Chen, J.-H. Jia, R. Yu, J.-Z. Liao, M.-X. Yang and C.-Z. Lu, Angew. Chemie Int. Ed., 2017, 56, 15006-15009.

X. Wang, S. Yang, Q. Tian, C. Zhong, Y. Qu, Y. Yu, Z. Jiang and L. Liao, Angew. Chemie Int. Ed., 2021, 60, 5213-5219.

X. Zheng, R. Huang, C. Zhong, G. Xie, W. Ning, M. Huang, F. Ni, F. B. Dias and C. Yang, Adv. Sci., 2020, 7, 1902087.

H. Miranda-Salinas, Y.-T. Hung, Y.-S. Chen, D. Luo, H.-C. Kao, C.-H. Chang, K.-T. Wong and A. Monkman, J. Mater. Chem. C, 2021, 9, 8819-8833.

K. Kawasumi, T. Wu, T. Zhu, H. S. Chae, T. Van Voorhis, M. A. Baldo and T. M. Swager, J. Am. Chem. Soc., 2015, 137, 11908-11911. 
C. C. A. Voll, J. U. Engelhart, M. Einzinger, M. A. Baldo and T. M. Swager, European J. Org. Chem., 2017, 4846-4851.

Y. Wada, H. Nakagawa, S. Matsumoto, Y. Wakisaka and H. Kaji, Nat. Photonics, 2020, 14, 643-649.

P. Lei, S. Zhang, N. Zhang, X. Yin, N. Wang and P. Chen, ACS Omega, 2020, 5, 28606-28614.

P. D. Bartlett, M. J. Ryan and S. G. Cohen, J. Am. Chem. Soc., 1942, 64, 2649-2653.

K. Baumgärtner, M. Hoffmann, F. Rominger, S. M. Elbert, A. Dreuw and M. Mastalerz, J. Org. Chem., 2020, 85, 15256-15272.

N. Harada, H. Uda, K. Nakasuji and I. Murata, J. Chem. Soc. Perkin Trans. 2, 1989, 10, 1449-1453.

T. Nakazawa and I. Murata, J. Am. Chem. Soc., 1977, 99, 1996-1997.

N. Harada, Y. Tamai, Y. Takuma and H. Uda, J. Am. Chem. Soc., 1980, 102, 501-506.

H. -D Martin and B. Mayer, Angew. Chemie Int. Ed. English, 1983, 22, 283-314.

L. Lv, W. Sun, Z. Jia, G. Zhang, F. Wang, Z. Tan and L. Zhang, Mater. Chem. Front., 2020, 4, 35393545.

T. Kodama, Y. Hirao, T. Nishiuchi and T. Kubo, Chempluschem, 2017, 82, 1006-1009.

T. Kodama, S. Miyazaki and T. Kubo, Chempluschem, 2019, 84, 1-5.

S. Montanaro, D. G. Congrave, M. K. Etherington and I. A. Wright, J. Mater. Chem. C, 2019, 7, 12886-12890.

L. Yu, Z. Wu, C. Zhong, G. Xie, Z. Zhu, D. Ma and C. Yang, Adv. Opt. Mater., 2017, 5, 1700588.

T. Huang, D. Liu, J. Jiang and W. Jiang, Chem. - A Eur. J., 2019, 25, 10926-10937.

P. Rajamalli, D. Chen, S. M. Suresh, Y. Tsuchiya, C. Adachi and E. Zysman-Colman, European J. Org. Chem., 2021, 2021, 2285-2293.

K. Zhang, F. Yang, Y. Zhang, Y. Ma, J. Fan, J. Fan, C.-K. Wang and L. Lin, J. Phys. Chem. Lett., 2021, 12, 1893-1903.

U. Balijapalli, R. Nagata, N. Yamada, H. Nakanotani, M. Tanaka, A. D’Aléo, V. Placide, M. Mamada, Y. Tsuchiya and C. Adachi, Angew. Chemie Int. Ed., 2021, 60, 8477-8482.

P. Meti, H. H. Park and Y. D. Gong, J. Mater. Chem. C, 2020, 8, 352-379.

L. Salah, M. K. Etherington, A. Shuaib, A. Danos, A. A. Nazeer, B. Ghazal, A. Prlj, A. T. Turley, A. Mallick, P. R. McGonigal, B. F. E. Curchod, A. P. Monkman and S. Makhseed, J. Mater. Chem. C, 2021, 9, 189-198.

T. M. Long and T. M. Swager, Adv Mater, 2001, 601-604.

S. Grimme, J. Antony, S. Ehrlich and H. Krieg, J. Chem. Phys., 2010, 132, 154104. 515-524.

55 T. Lu and F. Chen, J. Comput. Chem., 2011, 33, 580-592.

W. Zeng, T. Zhou, W. Ning, C. Zhong, J. He, S. Gong, G. Xie and C. Yang, Adv. Mater., 2019, 1901404.

57 W. Zhuang, S. Wang, Q. Tao, W. Ma, M. Berggren, S. Fabiano, W. Zhu and E. Wang, Macromolecules, 2021, 54, 970-980. 
59 P. Data, P. Pander, M. Okazaki, Y. Takeda, S. Minakata and A. P. Monkman, Angew. Chemie Int. Ed., 2016, 55, 5739-5744.

60 R. Pashazadeh, P. Pander, A. Lazauskas, F. B. Dias and J. V. Grazulevicius, J. Phys. Chem. Lett., 2018, 9, 1172-1177. 